\title{
Composição química dos óleos essenciais de Schinus molle e atividade antifúngica em Sclerotinia sclerotiorum
}

Antonio Carlos Pereira de Menezes Filho, Wendel Cruvinel de Sousa, Carlos Frederico de Souza Castro

Instituto Federal Goiano - IF Goiano, Mestrado em Agroquímica, Campus Rio Verde, GO. E-mail: astronomoamadorgoias@gmail.com

\section{Resumo}

Schinus molle é popularmente conhecida por aroeira-salsa, possuindo folhas e ramos aromáticos. 0 objetivo deste estudo foi avaliar a composição química dos óleos essenciais dos ramos e folhas de $S$. molle e a atividade antifúngica sobre o isolado de Sclerotinia sclerotiorum (mofo-branco). Os ramos e folhas foram coletados e processados para extração dos óleos essenciais por hidrodestilação em aparelho tipo Clevenger. Os rendimentos de extração de óleo essencial, e seus perfis químicos foram avaliados por cromatografia gasosa com espectrometria de massas. A atividade antifúngica foi determinada utilizando diferentes concentrações de ambos os óleos essenciais. A análise estatística foi realizada a partir das médias aritméticas, pelos testes de Student $(p \leq 0,05)$ e Scotti-Knott $5 \%$. Os rendimentos de óleo essencial foram de 0,21 e $0,37 \%$, com 16 e 21 compostos identificados para os óleos essenciais dos ramos e folhas, respectivamente. Os compostos majoritários foram $\delta$-cadieno $21,77 \%$, viridifloral $21,74 \%$, copaeno $12,9 \%$ e cariofileno 9,99\% para o óleo essencial dos ramos; para o óleo essencial das folhas foram, germacreno $D$ $39,00 \%$, $\alpha$-pineno $14,77 \%$, e germacreno B 6,25\%. A atividade antifúngica teve inibição micelial em $S$. sclerotiorum em todas as concentrações avaliadas, com destaque para $100 \mu \mathrm{L} \mathrm{mL}^{-1}$ que tiveram inibição de 86,4 e $81,3 \%$ para ramos e folhas, respectivamente. Os óleos essenciais de $\mathrm{S}$. molle tiveram bons rendimentos e são ricos em compostos monoterpênicos, sesquiterpênicos e possuem grande eficácia em atividade antifúngica.

Palavras-chave: Aroeira-salsa; Schinus; mofo-branco; compostos voláteis; perfil químico.

\section{Chemical composition from Schinus molle essential oils and antifungal acitivity over Sclerotinia sclerotiorum}

\begin{abstract}
Schinus molle is popularly known as aroeira-salsa, with aromatic leaves and branches. The present study was to evaluate the chemical composition of the essential oils from the branches and leaves from $S$. molle and, antifungal activity on the isolate from Sclerotinia sclerotiorum (white mold). The branches and leaves were collected and processed to extract essential oils by hydrodistillation in a Clevenger type apparatus. The yields from essential oils extraction, and their chemical profiles were evaluated by gas chromatography with mass spectrometry. The essential oils yields were 0.21 and $0.37 \%$, with 16 and 21 compounds identified for the essential oils of the branches and leaves, respectively. The major compounds were $\delta$ cadiene $21.77 \%$, viridiflorol 21.74 , copaene $12.9 \%$ and caryophyllene $9.99 \%$ for the essential oil of the branches, for essential oil of the leaves germacrene D 39.00\%, $\alpha$-pinene $14.77 \%$, and germacrene B $6.25 \%$. The antifungal activity had mycelial inhibition in S. sclerotiorum in all evaluated concentrations, with emphasis on $100 \mu \mathrm{L} \mathrm{mL}^{-1}$, which had inhibition from 86.4 and $81.3 \%$ for branches and leaves, respectively. The essential oils from $S$. molle had good yields and are rich in monoterpenics and sesquiterpenics compounds, and have great efficacy in antifungal activity.
\end{abstract}

Keywords: Aroeira-salsa; Schinus; white mold; volatile compounds; chemical profile. 


\section{Introdução}

Schinus molle L. conhecida popularmente como aroeira-salsa ou aroeira-periquita, pertence à família Anacardiaceae que inclui cerca de 30 espécies. S. molle é uma espécie nativa do Sul do Brasil, mas sua distribuição ocorre desde a América do Norte (México e Estados Unidos), América Central (El Salvador, Guatemala, Honduras) e parte da America do Sul (SANTOS et al., 2007; SANTOS et al., 2010; TLILI et al., 2018; SOLIS et al., 2019; ABOALHAIJA et al., 2019; REIBEIRO et al., 2019). Esta espécie possui propriedades medicinais com ações antiespasmódica, antirreumática, emenagoga, antinflamatória e cicatrizante, produzindo importantes metabólitos secundários como alcaloides, flavonoides, taninos e óleos essenciais (OEs) em suas folhas e ramos (MURRAY; MURRAY, 2017).

Os OEs são compostos naturais de alta complexidade e na maioria das vezes voláteis, sendo constituídos basicamente de monoterpenos, sesquiterpenos e fenilpropanóides. Apresentam papel importante no mecanismo de defesa e de reprodução dos vegetais, contra ataques a fitopatógenos, herbivoria e a insetos, possuindo também várias ações biológicas, como antifúngica, bactericida, antiviral, inseticida, larvicida, moluscicida, dentre outras (PEREIRA et al., 2016; BEKTAŞ et al., 2016; TOMAZONI et al., 2017; SCHWENGBER et al., 2017).

Os OEs de S. molle tem importante ação de inibição no processo de germinação, na proteção contra herbívoros, na atração de insetos polinizadores e na proteção contra o déficit hídrico (RIBEIRO et al., 2019; SIMÕES; SPITZER, 2000). Os OEs de $S$. molle têm atividade inseticida, antimicrobiana, repelente, carrapaticida, fungistática, antitumoral, antioxidante e antidepressivo (SANTOS et al., 2010; AVELAR et al., 2016; TOMAZONI et al., 2017; ABOALHAIJA et al., 2019). O ataque por fungos em culturas como a soja causam grandes prejuízos nas safras anualmente, sendo um dos maiores problemas enfrentados pelos produtores rurais. O fungo Sclerotinia sclerotiorum conhecido por mofo-branco, ocasiona lesões moles, acastanhadas, nos órgãos vegetativos da planta, sendo facilmente identificado pelo micélio de cor branca como algodão, que recobre as partes do tecido danificado (SILVA et al., 2018).
O S. sclerotiorum possui como forma de resistência estruturas conhecida por escleródios que permanecem viáveis por mais de 11 anos no solo (JULIATTI et al., 2013). Os fungicidas tradicionais com formulação química sintética conhecida, não são totalmente eficazes, causando a inviabilidade desses escleródios que ficam sob ou internamente no solo (GRABICOSKI et al., 2010). Entretanto, o uso de fungicidas sintéticos apresentam sérios problemas de âmbito toxicológico para as formas de vida da fauna local, solo, água e para os humanos (XIE et al., 2011; NIU et al., 2011; SILVA et al., 2018).

Estudos recentes com o uso de OEs vêm demonstrando bons resultados no controle e inviabilidade de inúmeras espécies fúngicas causadoras de fitopatologias em inúmeros vegetais de interesse agrícola como milho, soja, grãos de bico e diversos legumes, sendo importante que novos estudos sejam realizados em condições locais, avaliando os OEs de essências nativas como alternativa natural no combate fúngico. Pontos importantes que deve se considerar na avaliação de compostos químicos voláteis de plantas nativas é a facilidade de aquisição, baixo custo de produção e ausência de problemas ocasionados por produtos químicos sintéticos ao solo, ar, água e as diversas formas de vida como os insetos polinizadores (KUMAR et al., 2014; BONFIN, et al., 2015; SILVA et al., 2018).

O objetivo deste estudo foi avaliar a composição química dos óleos essenciais dos ramos e folhas e a atividade antifúngica de $S$. molle sobre $S$. sclerotiorum (mofo-branco).

\section{Material e Métodos}

Ramos e folhas de $S$. molle foram coletadas na área do Instituto Federal Goiano, Campus Rio Verde, no município de Rio Verde, estado de Goiás, Brasil, $\left(17^{\circ} 48^{\prime} 46.8^{\prime \prime}\right.$, $50^{\circ} 53^{\prime} 55.1^{\prime \prime} \mathrm{W}$ ), em agosto de 2019 , nas primeiras horas da manhã entre 6-8 h. Uma exsicata foi herborizada, devidamente identificada e registrada no Herbário do Instituto Federal Goiano, com o voucher HRV 1210. $100 \mathrm{~g}$ de material vegetal foram triturados em processador doméstico (Philco, Mod. PH900) com $500 \mathrm{~mL}$ de água destilada. Um aparelho de destilação tipo Clevenger (Marconi, Mod. MA553/2000) foi usado para a extração dos óleos essenciais com tempo de extração de 3,5 horas. O hidrolato foi coletado e transferido para um funil de separação 
de $500 \mathrm{~mL}$ (Laborglas) sendo lavado com 3 partes de diclorometano (30 $\mathrm{mL}$ para cada lavagem) (Alphatec, P.A - ACS, pureza 99,8\%). A fase orgânica foi reunida em um béquer de $250 \mathrm{~mL}$ (Laborglas) onde foi adicionado sulfato de sódio anidro (Fmaia, P.A - AS, pureza 98.9\%), e logo após, filtrado em papel de filtro quantitativo (Unifil, C42). O solvente foi evaporado em local escuro em ambiente com temperatura controlada de $20{ }^{\circ} \mathrm{C}$. O rendimento de $\mathrm{OE}$ foi determinado em porcentagem de extração conforme equação 1 , a seguir.

$\operatorname{Red} \%=\left(O E_{\text {extraído }} /\right.$ massa $\left._{\text {in natura }}\right) * 100 \quad$ Eq. [1]

O perfil químico do OE para ramos e

folhas de $S$. molle foi observado em sistema de cromatógrafo a gás com espectrômetro de massas (CG-EM). O CG-EM estava equipado com auto-injetor, coluna ( $30 \mathrm{~m} \times 0,250 \mathrm{~mm} \times 0,25 \mu \mathrm{m})$ fundida com sílica e espectrômetro de massas com detector por ionização por impacto eletrônico (II) (70 e.V) (PerkinElmer) com software TurboMass. A temperatura inicial foi mantida a $60{ }^{\circ} \mathrm{C}$ por 3 min., seguido de um acréscimo de $3{ }^{\circ} \mathrm{C} \mathrm{min.}{ }^{-1}$ até atingir $200{ }^{\circ} \mathrm{C}$, e posteriormente foi programada para um aumento de temperatura de $15^{\circ} \mathrm{C} \mathrm{min} .^{-1}$ até 280 ${ }^{\circ} \mathrm{C}$, permanecendo nessa temperatura por mais 1 $\min$.

As temperaturas do injetor e do detector foram de $230{ }^{\circ} \mathrm{C}$ e $300{ }^{\circ} \mathrm{C}$, respectivamente. As analises foram realizadas utilizando gás He como carreador com pressão de injeção de $57,4 \mathrm{KPa}$, razão de Splitless: 150, faixa de detecção do espectrômetro de massas: $43-550 \mathrm{~m} / \mathrm{z}$, start time (cut time do solvente): $3 \mathrm{~min}$. e fluxo de $3 \mathrm{~mL}$ $\min ^{-1}$. A identificação dos compostos do OE foi baseada no índice de retenção linear (Índice de Kovats) (IK) calculado em relação aos tempos de retenção da série homóloga de $n$-alcanos (C-12 a C-40) (Sigma-Aldrich) e no padrão de fragmentação observado nos espectros de massas, por comparação destes com a literatura (ADAMS, 2007) e da espectroteca (Nist 11) do CG-EM. A metodologia de injeção e análise dos OEs foi criada com padrões internos adotados.

$\mathrm{O}$ isolado de $\mathrm{S}$. sclerotiorum foi obtido a partir de escleródios formados no interior da haste de soja (Glycine max L.), proveniente de campos comerciais no município de Rio Verde, Goiás, Brasil. A cultura mantida em meio batata, dextrose e ágar (KASVI - BDA) foi doada pelo laboratório de Produtos Naturais do Instituto Federal Goiano, Campus Rio Verde, e mantido no banco micológico do laboratório de Química Tecnológica da mesma instituição.

A avaliação antifúngica dos OEs de $S$. molle sobre o crescimento micelial do isolado de S. sclerotiorum, foi realizada pelo método de difusão em placa, onde ocorreu em diferentes concentrações de $\mathrm{OE}$ testado, partindo de 100 (óleo puro); 50; 25; 12,5; 6,25; 3,13 e 1,56 $\mathrm{L} \mathrm{mL}^{-1}$ em diluições respectivas em dimetilsulfóxido (DMSO) (Vetec, P.A - ACS, pureza, 99\%). Como controles negativo, utilizou-se a testemunha (ausência de óleo essencial) e DMSO, e como controle positivo $\mathrm{O}$ fungicida comercial Frowncide $^{\circledR} 500$ SC (ISK, Fluazinam, conc. comercial $500 \mathrm{~g} \mathrm{~L}^{-1}$ ), registro no Ministério da Agricultura 7695, na concentração prédeterminada de $10 \mu \mathrm{LL}^{-1}$.

As concentrações do OE foram adicionadas ao meio de cultura BDA após esterilização e resfriamento, bem como para os tratamentos com fungicida comercial e DMSO. Após solidificação do meio, em câmara de fluxo laminar (Solab, Mod. PSRFLV-656/3), 1 disco de micélio de $S$. sclerotiorum com $7 \mathrm{~mm}$ de diâmetro, foi depositado no centro da placa de Petri (Laborglas) de $10 \mathrm{~cm}$ de diâmetro. Em seguida foram incubadas em estufa bacteriológica (SolidSteel, Mod. SSD) à temperatura entre $20-22{ }^{\circ} \mathrm{C}$, durante 48 horas (GARCIA et al., 2012, adaptado).

A avaliação consistiu em medições diárias do diâmetro das colônias, por meio de um paquímetro digital (Digimess, Mod. 100.170) 150 $\mathrm{mm} / 6^{\prime \prime}$ resolução $0,01 \mathrm{~mm}$, iniciadas após 24 horas do início da incubação e encerradas com tempo de 48 horas, quando as colônias fúngicas, do tratamento testemunha, atingiram completamente a área interna da placa de Petri. A determinação da porcentagem de inibição de crescimento (PIC), foi realizada conforme equação 2, a seguir, proposta por Garcia et al. (2012).

$$
\mathrm{PIC}=(\mathrm{DTT}-\mathrm{DTQ}) / \mathrm{DTT} \times 100 \quad \text { Eq. [2] }
$$

Onde: $\mathrm{PIC}=$ porcentagem de inibição do crescimento, DTT = diâmetro no tratamento testemunha, DTQ = diâmetro no tratamento químico.

A análise estatística consistiu através de triplicatas para determinação do rendimento de $\mathrm{OE}$, e de quadruplicata para o teste fúngico, seguido de $( \pm)$ desvio padrão das médias. Os dados foram submetidos à análise de variância (ANOVA) e as médias de todos os tratamentos com $\mathrm{OE}$, foram comparadas ao fungicida 
comercial pelo Teste de Scott-Knott, com nível de significância de $5 \%$. Já as médias entre os rendimentos foram avaliadas pelo Teste de Student $(p \leq 0,05)$. O Software estatístico utilizado foi o PAST 3 (versão livre 2019).

\section{Resultados e Discussão}

O rendimento dos OEs para ramos e folhas de $S$. molle foi de $0,21 \pm 0,15^{\text {a }}$ e de $0,37 \pm$ $0,06^{\mathrm{a}} \%$, respectivamente. Prado et al. (2019), encontraram rendimento de 1,2\% para o $\mathrm{OE}$ das folhas de $S$. molle. Nos estudos de El-Sayed et al. (2017), os pesquisadores relatam em estudo a produção de $1,87 \mathrm{~mL}$ de $\mathrm{OE}$ extraído do órgão foliar de S. molle coletados na região de Taif, Arábia Saudita. Tomazoni et al. (2017), encontraram para S. molle e S. terebinthifolius outra espécie do gênero Schinus, conteúdo de OE das folhas secas de 1,02 e 0,45\%. Já Vicenço et al. (2017), encontraram teor de OE para as folhas de S. molle igual a 1,16\%. E para Schinus aroeira, Solis-Quispe et al. (2016), encontraram rendimento de $0,15 \%$ para o $\mathrm{OE}$ das folhas.

Os compostos majoritários no $\mathrm{OE}$ dos ramos foram $\delta$-cadieno com $21,77 \%$, viridifloral $21,74 \%$, copaeno $12,9 \%$ e cariofileno com $9,99 \%$, e para $\mathrm{O} O \mathrm{OE}$ das folhas, destacaram-se os compostos germacreno D 39,00\%, a-pineno 14,77\%, e germacreno B com 6,25\%, (Tabela 1).
Tabela 1. Composição química dos óleos essenciais dos ramos (OER) e folhas (OEF) de $S$. molle.

\begin{tabular}{|c|c|c|}
\hline \multicolumn{3}{|c|}{ OER } \\
\hline Composto $^{a}$ & IR & \% ÁREA \\
\hline & & RELATIVA* \\
\hline Linalol & 053 & 3,25 \\
\hline Iso-citronelol & 199 & 0,37 \\
\hline$\beta$-mirceno & 988 & 0,22 \\
\hline$\beta$-citral & 1244 & 0,11 \\
\hline Geraniol & 1257 & 5,21 \\
\hline$\delta$-eleneno & 1298 & 6,27 \\
\hline Copaeno & 1339 & 12,9 \\
\hline$\beta$-eleneno & 1356 & 0,95 \\
\hline Cariofileno & 1384 & 9,99 \\
\hline$\beta$-copaeno & 1443 & 0,85 \\
\hline Germacreno B & 1464 & 0,46 \\
\hline$\beta$-selinene & 1490 & 4,14 \\
\hline$\delta$-cadieno & 1491 & 21,77 \\
\hline Viridifloral & 1550 & 21,74 \\
\hline Cubenol & 1583 & 0,97 \\
\hline$(Z, E)$-Farnesol & 1634 & 1,85 \\
\hline Total & & $91,05 \%$ \\
\hline \multicolumn{3}{|c|}{ OEF } \\
\hline$\alpha$-pineno & 938 & 14,77 \\
\hline Canfeno & 954 & 1,84 \\
\hline Mirceno & 992 & 0,59 \\
\hline ס-3-careno & 1012 & 0,73 \\
\hline$\gamma$-terpineno & 1062 & 0,68 \\
\hline Mirtenol & 1198 & 0,23 \\
\hline Acetato de bornila & 1291 & 5,33 \\
\hline Acetato de nerila & 1371 & 3,58 \\
\hline$\beta$-bourboneno & 1388 & 4,52 \\
\hline$\beta$-curjuneno & 1434 & 0,55 \\
\hline$\beta$-Aromadendreno & 1443 & 0,21 \\
\hline Allo-aromadendreno & 1460 & 2,00 \\
\hline Germacreno D & 1484 & 39,0 \\
\hline Cubebol & 1518 & 0,75 \\
\hline Germacreno B & 1561 & 6,25 \\
\hline Espatulenol & 1581 & 3,33 \\
\hline Globulol & 1585 & 3,21 \\
\hline$\alpha$-acorenol & 1632 & 2,34 \\
\hline$\alpha$-cadinol & 1654 & 0,54 \\
\hline Valeraniol & 1660 & 0,22 \\
\hline $\begin{array}{l}\text { Óxido } \\
\text { aromandendreno }\end{array}$ & 1692 & 2,01 \\
\hline Total & & $92,68 \%$ \\
\hline
\end{tabular}


Um total de 16 compostos foi identificado para o $\mathrm{OE}$ dos ramos e 21 compostos para o OE das folhas de S. molle. Ambos os óleos essenciais apresentam conteúdo complexo de compostos monoterpênicos e sesquiterpenos.

Prado et al. (2019), encontraram 12 compostos no OE de $S$. molle, sendo o $\beta$-pineno $25,23 \%$, Epi- $\alpha$-cadinol 21,29\%, $\alpha$-pineno 18,72, mirceno $11,54 \%$ e sabineno com 5,02\% como compostos majoritários. Nos estudos de Silva et al. (2019a), os pesquisadores encontraram para o $\mathrm{OE}$ das folhas de $S$. lentiscifolius outra espécie do gênero Schinus, os seguintes compostos majoritários $\delta$-cadieno $14,4 \%$, limoneno $8,14 \%$, sabineno $5,08 \%, \alpha$-cadinol $4,91 \%, \alpha$-pineno $4,80 \%$ e para terpinen-4-ol com 3,85\%.

Santos et al. (2007), obtiveram perfil químico do $\mathrm{OE}$ das folhas de $S$. molle com 28 compostos identificados, sendo os majoritários $\alpha$ pineno, $\beta$-pineno, mirceno, limoneno, $\beta$ cariofileno, biciclogermacreno, $\delta$-cadieno, espatulenol e $\alpha$-cadinol. Vicenço et al. (2017), encontraram como composto majoritário apenas o a-pineno com $44 \%$ para as folhas de $S$. molle. Tomazoni et al. (2017), encontraram como compostos majoritários $\alpha$-pineno 25,33 e 27,85\%, e para $\beta$-pineno 24,05 e $8,37 \%$ para os OEs das folhas de $S$. molle e $S$. terebinthifolius, respectivamente. Já nos estudos de Solis-Quispe et al. (2016), avaliando o OE das folhas de $S$. aroeira, os pesquisadores encontraram os seguintes compostos majoritários canfeno $8,4 \%$, $\alpha$-felandreno $18,2 \%$, e limoneno 9,4\%.

Quando comparado à outra espécie de Schinus, $S$. terebinthifolius os compostos majoritários do OE das folhas em ambos os gêneros (macho e fêmea) foram $\delta$-3-careno $27,8 \%$ e $\beta$-cariofileno com $30,2 \%$, e para o perfil químico do $\mathrm{OE}$ macho apresentou o D-limoneno com $96,6 \%$ sendo o composto majoritário (SILVA et al., 2019b). Observa-se neste, e nos estudos discutidos, que a variação química apresenta grande diferentes proporções para os constituintes de $S$. molle, visto que, os estudos foram realizados em diferentes regiões apresentando uma gama de compostos e teores, o mesmo é discutido nos estudos de Aboalhaija et al. (2019), em estudos com esse vegetal na Turquia (BASER et al., 1997; DEVECl et al., 2010; ERYIGIT et al., 2017).

Isso pode ser devido a fatores como discutido por El-Sayed et al. (2017), onde a variação nos constituintes e seus teores em $S$. molle, têm como consequência as variações biológicas para diferentes localidades, alta da área de coleta em relação ao nível do mar, pluviosidade, clima, solo, nutrientes do solo, estresse em que a planta passou ou está passando no momento das coletas de material, bem como a genética dos grupos de indivíduos vegetais em que foram analisados (quimiotipo). Outro ponto importante na avaliação de rendimento, é as condições em que o vegetal é submetido, Ribeiro et al. (2019), submeteram plantas de $S$. molle em concentrações de $\mathrm{Pb}\left(\mathrm{NO}_{3}\right)_{2}$ por 90 dias, e em seguida determinaram o teor de OE com rendimento de $0,0083 \%$.

A porcentagem de inibição de crescimento em ambos os óleos essenciais de $S$. molle tem boa efetividade inibindo o crescimento de $S$. sclerotiorum nas diversas concentrações testadas, em especial para a concentração $100 \mu \mathrm{L}$ $\mathrm{mL}^{-1}$, apresentando inibição de 86,4 e $81,3 \%$ para os OEs dos ramosos e folhas, respectivamente (Figura 1). 
Figura 1. Percentual de inibição de crescimento PIC para o isolado de Sclerotinia sclerotiorum em diferentes concentrações dos $\mathrm{OEs}$ de $S$. molle. Médias seguidas de mesma letra para cada OE não diferem entre si pelo teste de Scott-Knott com nível de significância de 5\%.

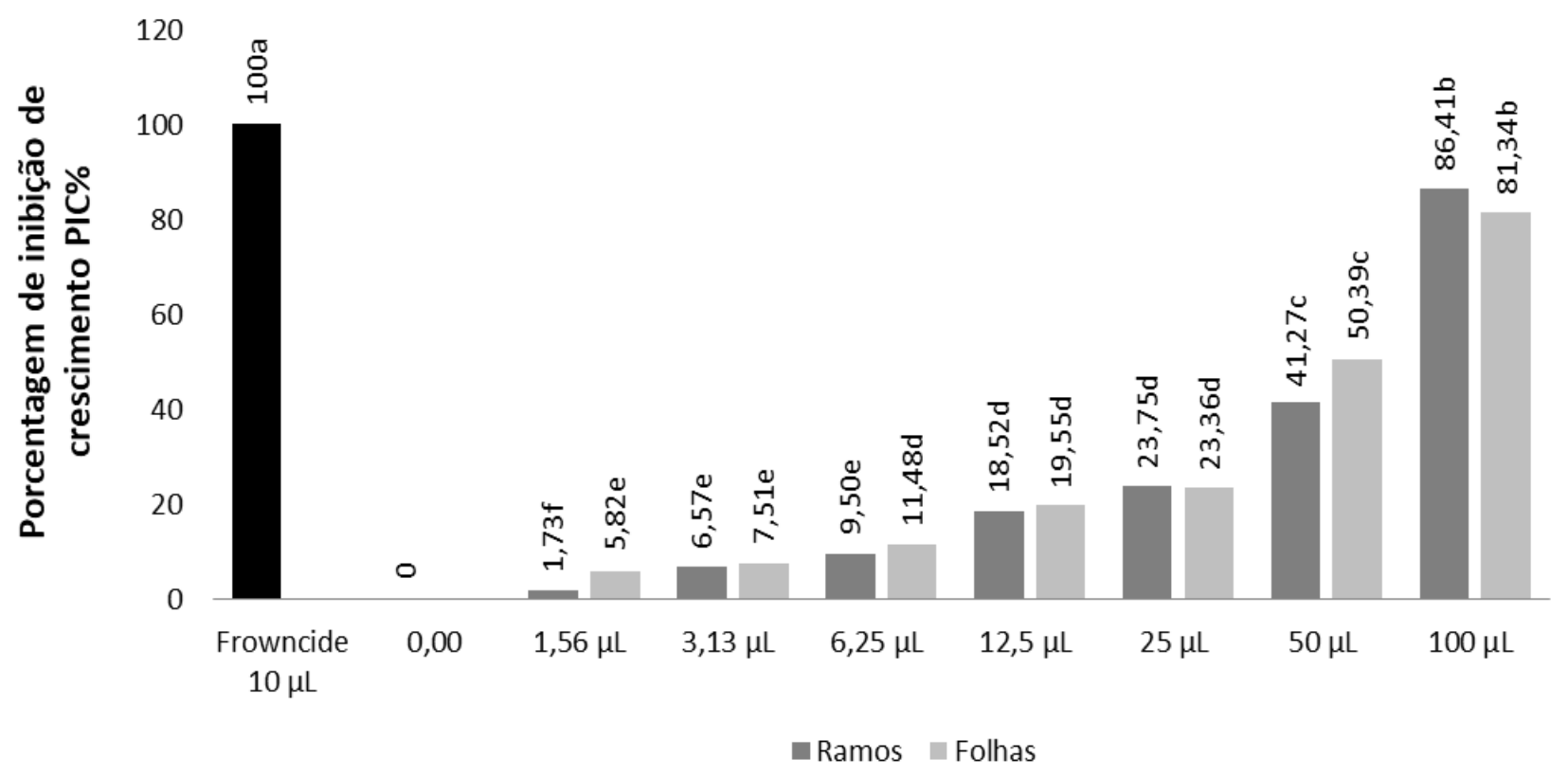

Em todas as concentrações em ambos os OEs houve ação de inibição no desenvolvimento micelial de $S$. sclerotiorum. Os OEs dos ramos e folhas apresentaram na maior concentração de $100 \mu \mathrm{L} \mathrm{mL}{ }^{-1}$ as maiores taxas de PIC conforme o teste de Scott-Knott 5\% de significância, diferindo do fungicida comercial Frowncide 500 SC que apresentou taxa de PIC de $100 \%$.

Deste modo, a maior concentração de ambos os OEs mostram ser mais eficazes dentre as demais concentrações avaliadas para S. molle. Os OEs extraídos dos ramos e folhas de $S$. molle tiveram bons resultados como agentes fungitóxicos, sendo uma opção fitofúngica sobre ao isolado de $S$. sclerotiorum. A atividade antifúngica para os OEs dos ramos e folhas estão relacionados aos compostos voláteis dos OEs, que provavelmente atuam por sinergismo provocando sensibilidade nas hifas de $S$. sclerotiorum, inibindo o seu desenvolvimento.

Tomazoni et al. (2017), observaram alta eficiencia de inibição de crescimento em Alternaria solani com $59,46 \%$, Septoria lycopersici de $60,42 \%$ e para Stemphylium solani com $66,13 \%$ em isolados a partir de tomateiros, em uma concentração de $20 \mu \mathrm{L} \mathrm{m}^{-1}$ de OE das folhas de $S$. molle. Os pesquisadores ainda obtiveram importante taxa de PIC avaliando o OE das folhas de S. terebinthifolius com $54,21 \%$ para $A$. solani, $41,06 \%$ para $S$. lycopersici e de $57,6 \%$ para $S$. solani. Vicenço et al. (2017), não encontraram taxas de inibição sobre o isolado de Cercospora kikuchii para os OEs das folhas de S. molle e $S$. terebinthifolius mesmo na concentração máxima de $0,20 \%$.

Nos estudos de Xavier et al. (2016), os investigadores avaliaram a ação fungicida do $\mathrm{OE}$ das folhas de Cardiopetalum calophyllum sobre o isolado de $S$. sclerotiorum onde obtiveram máxima inibição micelial de $87,63 \%$, próximo ao deste estudo. Os autores complementam que, tal inibição possivelmente está relacionada ao composto espatulenol que no estudo desenvolvido por esses pesquisadores, apresenta como composto majoritário, ou também pelo sinergismo dos compostos presentes no $\mathrm{OE}$, carecendo de novas pesquisas, possivelmente isolando os compostos e testando novamente in vitro. Já Santos et al. (2010), onde avaliaram o OE das folhas de $S$. molle encontraram doses letais para Botrytis spp. e Fusarium spp., com diluições inferiores a $10 \%$. No mesmo estudo, os autores observaram que, a letalidade para o gênero Alternaria spp. e Colletotrichum spp., ocorrem em diluição de OE igual ou superior a $25 \%$.

\section{Conclusão}

Os óleos essenciais dos ramos e folhas de S. molle obtidos por hidrodestilação tiveram bom rendimento de produção, riqueza no perfil químico volátil, e como agente antifúngico in vitro no controle de Sclerotinia sclerotiorum. 
Como observado, os óleos de S. molle, são eficientes para uso futuro em novos experimentos no controle de $S$. sclerotiorum in vivo, (casa-de-vegetação, em campo de culturas, e incorporado à micro ou nanoesferas de quitosana) para que se possa comparar entre os resultados obtidos neste estudo.

\section{Agradecimentos}

Ao Instituto Federal de Educação, Ciência e Tecnologia Goiano, Campus Rio Verde; a Universidade Federal de Jataí, Campus Jatobá; a Universidade Federal de Goiás, Campus Samambaia; aos laboratórios de Sistemática Vegetal, Microbiologia de Alimentos, Química Tecnológica; Águas e Efluentes, Química de Produtos Naturais, e de Bioensaios e Biomoléculas; a Central Analítica Multi-Usuário CEMA; ao Herbário do Instituto Federal Goiano, Campus Rio Verde.

\section{Referências}

ABOALHAIJA, N. H.; AWWAD, O.; KHALIL, E.; ABBASSI, R.; ABAZA, I. F.; AFIFI, F. U. Chemodiversity and antiproliferative activity of the essential oil of Schinus molle L. growing in Jordan. Chemistry \& Biodiversity, v. 16, n. 11, p. 1-11, 2019.

https://doi.org/10.1002/cbdv.201900388

ADAMS, R. P. Identification of essential oils components by gas chromatography/mass spectrometry. 4th. ed. Carol Stream, IL: I Allured Publ. Corp., $2007 . \quad 804 \quad$ p.

AVELAR, B. R. de; LAMBERT, M. M.; SANTOS SIQUEIRA, R. C.; CID, Y. P.; CHAVES, D. S. A.; COUMENDOUROS, K. Atividade in vitro de óleos essenciais e extratos de Schinus molle L. frente à Rhipicephalus microplus. Revista Brasileira de Medicina Veterinária, v. 38, (Supl. 3), p. 183-187, 2016.

http://rbmv.org/index.php/BJVM/article/view/30 $\underline{5}$

BASER, K.; KÜRKÇÜOGLU, M.; DEMIRÇAKMAK, B.; UÜLKER, N.; BEIS, S. H. Composition of the essential oil of Schinus molle L. grown in Turkey. Journal of Essential Oil Research, v. 9, n. 6, p. 693-696, 1997.

https://doi.org/10.1080/10412905.1997.9700813

BEKTAŞ, E.; DAFERERA, D.; SÖKMEN, M.; SERDAR, G.; ERTÜRK, M.; POLISSIOU, M. G.; SÖPKMEN, A.
In vitro antimicrobial, antioxidant, and antiviral activities of the essential oil and various extracts from Thymus nummularis M. Bieb. Indian Journal of Traditional Knowledge, v. 15, n. 3, p. 403-410, 2016. http://hdl.handle.net/123456789/34276

BONFIN, N. S.; NAKASSUGI, L. P.; OLIVEIRA, J. F. P.; KOHIYAMA, C. Y.; MOSSINI, S. A. G.; GRESPAN, R.; NERILO, S. B.; MALLMAN, C. A.; ABREU FILHO, B. A.; MACHINSKI JÚNIOR, M. Antifungal activity and Inhibition of fumonisin production by Rosmarinus officinalis L. essential oil in Fusarium verticillioides (Sacc.) Nirenberg. Food Chemistry, v. 166, n. 1, p. 330-336, 2015.

https://doi.org/10.1016/j.foodchem.2014.06.019

DEVECI, O.; SUKAN, A.; TUZUN, N.; KOCABAS, E. E. $\mathrm{H}$. Chemical composition, repellent and antimicrobial activity of Schinus molle L. Journal of Medicinal Plants Research, v. 4, n. 21, p. 22112216, 2010.

EL-SAYED, S.; ABDEL-HAMEED, BAZAID, S. A. Chemical composition of essential oils from leaves of Schinus molle L. growing in Taif, KSA. Journal of Essential Oil Bearing Plants, v. 20, n. 1, p. 45-58, 2017.

https://doi.org/10.1080/0972060X.2017.1294999

ERYIGIT, T.; YILDIRIM, B.; EKICI, K.; ÇIRKA, M. Chemical composition, antimicrobial and antioxidante properties of Schinus molle $\mathrm{L}$. essential oil from Turkey. Journal of Essential Oil Bearing Plants, v. 20, n. 2, p. 570-577, 2017. https://doi.org/10.1080/0972060X.2017.1304286

GARCIA, R. Á.; JULIATTI, F. C.; BARBOSA, K. A. G.; CASSEMIRO, T. A. Atividade antifúngica de óleo e extratos vegetais sobre Sclerotinia sclerotiorum. Bioscience Journal, v. 28, n. 1, p. 48-57, 2012. http://www.seer.ufu.br/index.php/biosciencejou rnal/article/view/8174

GRABICOSKI, E. M.; JACCOUD-FILHO, D. S.; HENNEBERG, L.; VRISMAN, C. M.; MARTINS NETO, M. O. Potencial inibitório de extratos de plantas para Sclerotinia sclerotiorum. In: Congresso Brasileiro de Fitopatologia/Annual Meeting of the Brazilian Phytopathological Society, 43., 2010, Cuiabá. Resumos [...] Cuiabá, MT: Brazilian Phytopathological Society, 2010. p. 28-30.v. 5. 
JULIATTI, F. C.; CRATO, F. F.; JULIATTI, F. C.; COUTO, K. R.; JULIATTI, B. C. M. Escala diagramática para avaliação da severidade de mofo branco em soja. Bioscience Journal, v. 29, n. 3, p. 676-680, 2013.

http://www.seer.ufu.br/index.php/biosciencejou rnal/article/view/22440

KUMAR, V.; MATHELA, C. S.; TEWARI, G.; SINGH, D.; TEWARI, A. K.; BISHT, K.; BISHT, K. S. Chemical composition and antifungal activity of essential oils from three Himalayan erigeron species. LWT - Food Science and Technology, v. 56, n. 2, p. 278-283, 2014.

https://doi.org/10.1016/j.Iwt.2013.12.007

MURRAY, A. P.; MURRAY, M. G. Phytochemistry, traditional uses and bioactivity of the medicinal plant Schinus aroeira L. (Anacardiaceae): A review. The Natural Products Journal, v. 7, n. 2, p. 97-103, 2017.

http://dx.doi.org/10.2174/221031550766617011 7145728

NIU, B.; WANG, W.; XIE, X. Advance on the control of Sclerotinia sclerotiorum. Life Science Research, v. 16, n. 1, p. 537-540, 2011.

PEREIRA, K. C.; REDA, F. R.; PIVETA, G.; GARCIA, F. A. O. Avaliação de óleos essenciais na qualidade sanitária e fisiológica em sementes e mudas de Schinus molle. Pesquisa Florestal Brasileira, v. 36, n. 85, p. 71-78, 2016.

https://doi.org/10.4336/2016.pfb.36.85.905

PRADO, A. C.; GARCES, H. G.; BAGAGLI, E.; RALL, V. L. M.; FURLANETTO, A.; JÚNIOR, A. F.; FURTADO, F. B. Schinus molle essential oils as a potential source of bioactive compounds: antifungal and antibacterial properties. Journal os Applied Microbiology, v. 126, n. 2, p. 516-522, 2019. https://doi.org/10.1111/jam.14157

RIBEIRO, V. E.; PEREIRA, M. P.; CASTRO, E. M. de; CORRÊA, F. F.; CARDOSO, M. G.; PEREIRA, F. J. Enhanced essential oil and leaf Anatomy of Schinus molle plants under lead contamination. Industrial Crops \& Products, v. 132, p. 92-98, 2019.

https://doi.org/10.1016/j.indcrop.2019.02.014

SANTOS, A. C. A. dos; ROSSATO, M.; SERAFINI, L. A.; BUENO, M.; CRIPPA, L. B.; SARTORI, V. C.; DELLACASSA, E.; MOYNA, P. Efeito fungicida dos óleos essenciais de Schinus molle L. e Schinus terebinthifolius Raddi, Anacardiaceae, do Rio Grande do Sul. Brazilian Journal of Pharmacognosy, v. 20, n. 2, p. 154-159, 2010. http://dx.doi.org/10.1590/S0102695X2010000200003

SANTOS, R. M. dos; AGOSTINI, F.; DE ALMEIDA, M. L.; PAULETTI, G. F.; SERAFINI, L. A.; MOYNA, P.; DELLACASSA, E. Caracterização química de populações de Schinus molle L. do Rio Grande do Sul. Revista Brasileira de Biociências, v. 5, supl. 2, p. 1014-1016, 2007.

SCHWENGBER, R. P.; BORDIN, J. C.; BORTOLUCCI, W. C.; CARPI, M. C. G.; ANDRÉ, V. C. S.; PACCOLAMEIRELLES, L. D.; GOMES, S. M. S. Óleo essencial das folhas e frutos de Schinus terebinthifolius Raddi no controle de Pratylenchus zeae. Arquivos de Ciências Veterinárias e Zoologia da UNIPAR, v. 20, n. 3, p. 153-159, 2017.

https://doi.org/10.25110/arqvet.v20i3.2017.6692

SILVA, E. A. J.; SILVA, V. P.; ALVES, C. C. F.; ALVES, J. M.; SOUCHIE, E. L.; BARBOSA, L. C. A. Chemical composition of the essential oil of Psidium guajava leaves and its toxicity against Sclerotinia sclerotiorum. Semina: Ciências Agrárias, v. 39, n. 2, p. 865-874, 2018.

http://dx.doi.org/10.5433/1679-

0359.2018v39n2p865

SILVA, E. R. da; PAWLOWSKI, Â.; LAZAROTTO, D. C.; SOARES, G. L. G. Antioxidant activity of essential oils from leaves of Schinus lentiscifolius Marchand. Revista Cubana de Plantas Medicinales, v. 24, n. 2, p. 1-8, 2019a.

SILVA, P. T. da; AZEVEDO, F. R. P.; DIAS, F. M. F.; LIMA, M. C. L.; RODRIGUES, T. H. S.; DE SOUZA, E. B.; BANDEIRA, P. N.; SANTOS, H. S. Composição química do óleo essencial extraído das folhas dos indivíduos macho e fêmea e frutos de Schinus terebinthifolius. Revista Virtual de Química, v. 11 , n. 1, p. 180-189, 2019b.

https://doi.org/10.21577/1984-6835.20190013

SIMÕES, C. M. O.; SPITZER, V. Óleos voláteis. In: SIMÕES, C. M. O.; SCHENKEL, E. P.; GOSMANN, G.; MELLO, J. C. P.; MENTZ, L. A.; PETROVICK, P. R. Farmacognosia da planta ao medicamento. Porto Alegre: Editora da Universidade Federal do Rio Grande do Sul, 2000. p. 397-426. 
SOLIS, J. I.; DAVILLA, R.; SANDOVAL, C.; GUZMÁN, D.; ALEJO, L.; KIROS, Y. Ethanol production from Schinus molle essential oil extraction residues. Waste and Biomass Valorization, p. 1-13, 2019. https://doi.org/10.1007/s12649-019-00737-3

SOLIS-QUISPE, L.; TOMAYLLA-CRUZ, C.; CALLOCHOQUELVICA, Y.; SOLÍS-QUISPE, A.; RODEIRO, I.; HERNÁNDEZ, I.; FERNÁMDEZ, M. D.; PINO, J. A. Chemical composition, antioxidant and antiproliferative activities of essential oil from Schinus aroeira L. and Minthostachys spicata (Benth.) Epl. Grown in Cuzco, Peru. Journal of Essential oils Research, v. 28, n. 3, p. 234-240, 2016.

http://dx.doi.org/10.1080/10412905.2015.11206 $\underline{91}$

TLILI, N.; YAHIA, Y.; FERIANI, A.; LABIDI, A.; GHAZOUANI, L.; NASRI, N.; SAADAOUI, E.; KHALDI, A. Schinus terebinthifolius vs Schinus molle: a Comparative study of the effect of species and location on the phytochemical content of fruits. Industrial Crops \& Products, v. 122, n. 15, p. 559-565, 2018.

https://doi.org/10.1016/j.indcrop.2018.05.080

TOMAZONI, E. Z.; RIBEIRO, R. T. S.; SCHWAMBACH, J. Potencial fungitóxico dos óleos essenciais de Schinus molle L. e Schinus terebinthifolius Raddi contra fungos patogênicos do tomateiro. Revista Brasileira de Agroecologia, v. 12, n. 2, p. 1-7, 2017.

VICENÇO, C. B.; FELTRACCO, M. C.; CAVIÃO, H. C.; PANSERA, M. R.; SARTORI, V. C. Composição química e atividade in vitro de óleos essenciais sobre a mancha púrpura da soja. Revista Interdisciplinar e Ciência Aplicada, v. 2, n. 4, p. 15, 2017.

XAVIER, M. N.; ALVES, J. M.; CARNEIRO, N. S.; SOUCHIE, E. L.; SILVA, E. A. J.; MARTINS, C. H. G.; AMBROSIO, M. A. L. V.; EGEA, M. B.; ALVES, C. C. F.; MIRANDA, M. L. D. Composição química do óleo essencial de Cardiopetalum calophyllum Schltdl. (Annonaceae) e suas atividades antioxidante, antibacteriana e antifúngica. Revista Virtual de Química, v. 8, n. 5, p. 1-16, 2016.

https://doi.org/10.21577/1984-6835.20160101

XIE, J.; XIAO, X.; FU, Y.; LIU, H.; CHENG, J.; GHABRIAL, S. A.; LI, G.; JIANG, D. A novel mycovirus closely related to hypoviruses that infects the plant pathogenic fungus Sclerotinia sclerotiorum. Virology, v. 418, n. 1, p. 49-56, 2011. https://doi.org/10.1016/j.virol.2011.07.008 